\title{
An Unusual Case of Infantile Spasms Due to a Pathogenic Variant in the MECP2 Gene
}

\author{
Behshad Charkhand ${ }^{1,2}$ Natarie Liu ${ }^{3}$ Karlene T. Barrett ${ }^{1,2}$ Walla Al-Hertani ${ }^{1,4}$ \\ Morris H. Scantlebury ${ }^{1,2,3}$
}
${ }^{1}$ Department of Pediatrics, Cumming School of Medicine, University of Calgary, Calgary, Alberta, Canada
2 Alberta Children's Hospital Research Institute, University of Calgary, Calgary, Alberta, Canada
3 Department of Clinical Neurosciences, Cumming School of Medicine University of Calgary, Calgary, Alberta, Canada
${ }^{4}$ Department of Medical Genetics, Cumming School of Medicine,
University of Calgary, Calgary, Alberta, Canada

Address for correspondence Morris H. Scantlebury, MD, Department of Paediatrics, Alberta Children's Hospital Research Institute, Cumming School of Medicine, University of Calgary, 278 HMRB, 3330 Hospital Drive, Calgary, Alberta, T2N 4N1, Canada

(e-mail: morris.scantlebury@albertahealthservices.ca).

J Pediatr Neurol 2020;18:39-44.

\begin{abstract}
Keywords

- epileptic encephalopathy

- Rett's syndrome

- cardiomyopathy

The infantile spasms (IS) syndrome is a developmental epileptic encephalopathy disorder characterized by epileptic spasms occurring in infancy, hypsarrhythmia on the electroencephalography (EEG) and developmental arrest or regression. The etiologies include structural, metabolic, and genetic causes. We report an unusual case of IS due to a de novo variant in the MECP2 gene. The patient also had variants of uncertain significance in the SCN9A and SCN5A genes inherited from the father and mother, respectively. This report highlights the need for broad genetic testing in MECP2-related disorders with atypical presentations to better understand the disease etiology.
\end{abstract}

\section{Introduction}

The infantile spasms (IS) syndrome is an age-dependent epileptic encephalopathy spectrum disorder characterized by flexion, extension, mixed or subtle spasms that are usually brief and can occur multiple times daily, often clustering around the sleep-wake transition. The ictal electroencephalography (EEG) pattern associated with the spasms is characterized by a high-voltage generalized discharge, followed by low amplitude fast activity, and voltage attenuation, known as the electrodecremental response. A chaotic, high-voltage background, with multifocal independent spikes termed hypsarrhythmia, which some consider a form of status epilepticus, is recorded interictally. In most patients, developmental arrest and regression usually ensues and current evidence supports that the earlier cessation of spasms and improvement of the interictal EEG (i.e., absence of hypsarrhythmia) the better the outcome with regards to the development of other seizure types and persistent developmental delays. Usual first-line treatments are either hormonal (adrenocorticotropic hormone [ACTH] or prednisolone) or antiepileptic (vigabatrin); however, they are not always effective. In refractory cases, alternative treatments include other antiseizure medications, such as valproate, topiramate, and the ketogenic diet amongst other treatments with variable efficacy. ${ }^{1}$

Infantile spasms are categorized into symptomatic IS and cryptogenic IS based on etiology. Symptomatic IS are defined as IS of unknown etiology with a normal magnetic resonance imaging (MRI) early on in the course of the disease, whereas cryptogenic IS exhibits normal MRI findings but the underlying cause is suspected to be genetic. ${ }^{2}$ With the continued advancement and expansion of genetic testing, new causative genes are constantly being identified in cases of cryptogenic IS. Mutations in genes concerning neuronal migration, synaptogenesis, normal receptor, and ionic-channel received

November 26, 2018

accepted after revision

February 8, 2019

published online

March 13, 2019
Copyright @ 2020 by Georg Thieme Verlag KG, Stuttgart · New York
DOI https://doi.org/ 10.1055/s-0039-1683436. ISSN 1304-2580. 
expression have been described. Some examples include mutations in the ARX, CDKL5, STXBP1, FOXG1, GRIN1, GRIN2A, and SLC25A22 genes. ${ }^{3}$ Here we describe an in-depth report of a case of IS due to a mutation in the MECP2 gene which has previously been described in patients with Rett's syndrome. ${ }^{4}$

\section{Case Report}

This patient is a 9-month-old girl of Lebanese decent who was brought to hospital because of a history of flexion spasms and focal to bilateral convulsive seizures starting at 7 and 9 months old, respectively. The patient also had episodic eye movements beginning at 3 months old, but no obvious seizures were reported. The patient was born at 41 weeks via Cesarian section for failure to progress. Mother was a 28-year-old prima gravida when the patient was born. There were no complications in the pregnancy. Development was reported as normal until 3 months of age when the parents noted that the patient was not fixing or tracking with her eyes. At the time of the visit, she had not yet learned to turn over or sit. She had never held a bottle. There was no pincer grasp bilaterally. She fixed and tracked inconsistently and did not smile reciprocally. There may have been a regression in her social skills (loss of fixing and smiling) with the spasms. On family history her great grandparents were both from Lebanon and were related. She had a distant relative (maternal cousin) who at the time of the visit was 35 years old and had epilepsy; no further details were available. There was no further contributory family history and the parents were both healthy. On examination, the patient was macrocephalic with a head circumference of $47 \mathrm{~cm}$ (97 percentile; - Fig. 1). Her general, cardiovascular, lung, abdomen, and skin examinations were normal. On neurological examination, she was awake and not inter-relative. She tracked slightly and inconsistently with her eyes. Her cranial nerve examination was normal. Fundoscopic examination, which was also confirmed by an ophthalmologist, was normal. On motor examination she had significant axial and appendicular hypotonia. She moves all limbs symmetrically but tended to fist her left more than her right hand. Her deep tendon reflexes were 1 and symmetric and her toes were downward going to bilateral plantar stimulation. No dysmetria was noted. Sensation to light touch was normal.

Video EEG recording showed the typical features of IS ( -Fig. 2A, B). MRI brain performed at 9 months showed slightly enlarged ventricles. Single voxel magnetic resonance spectroscopy (MRS) from the lateral ventricle showed a lactate doublet whereas MRS from the left basal ganglia was normal (-Fig. 3A, B). MRI brain at 3 months of age was normal. Extensive metabolic testing which included plasma and urine amino acids-pipecolic acid, organic acids were normal. Plasma, total and free carnitine levels, acylcarnitine profile, total homocysteine, and very long chain fatty acids were normal. Urine-uronic acids, 3-methylglutaconic acid, oligosaccharides, mucopolysaccharides, sulfites, purines, and pyrimidines were all normal. The enzymatic activities of galactocerebrosidase, $\beta$-glucosidase, hexosaminidase, arylsulfatase $A$ and biotinidase were all normal. Cerebrospinal fluid (CSF) studies including lactate and glucose were normal. No diagnostic inclusions of neuronal ceroid lipofuscinosis, or structurally abnormal mitochondria, or demonstrable lysosomal abnormalities, were detected in fibroblasts on electron microscopy. Visual evoked potentials showed normal conduction in the visual pathways bilaterally. No microdeletions or microduplications were identified on comparative genomic hybridization microarray. A genetic

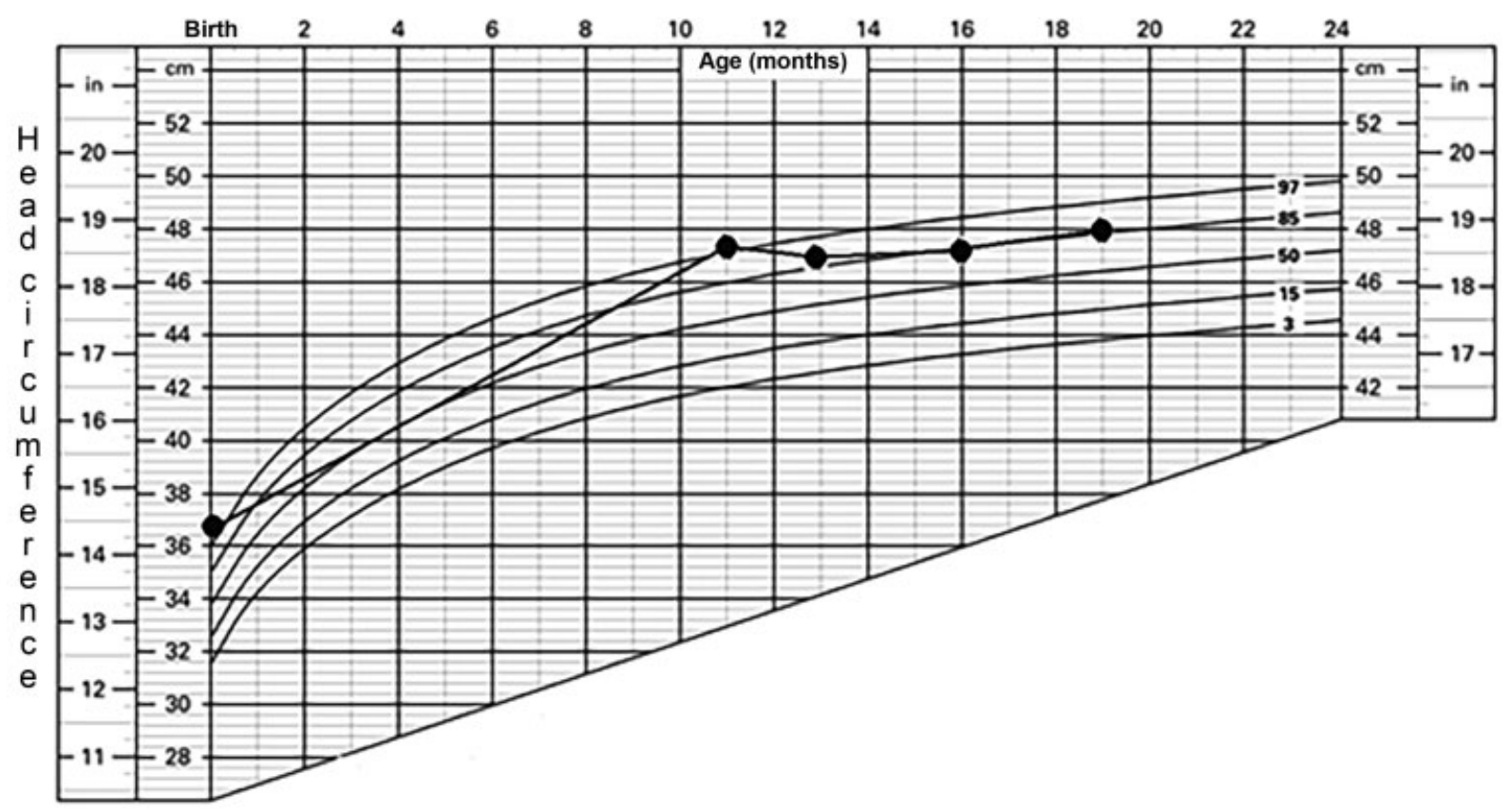

Fig. 1 Head circumferences of the patient with IS plotted on birth to 24 months WHO growth chart for Canada. Note the macrocephaly at birth and at 11 months with deceleration of head growth becoming apparent prior to her death at 19 months. IS, infantile spasms; WHO, World Health Organization. 


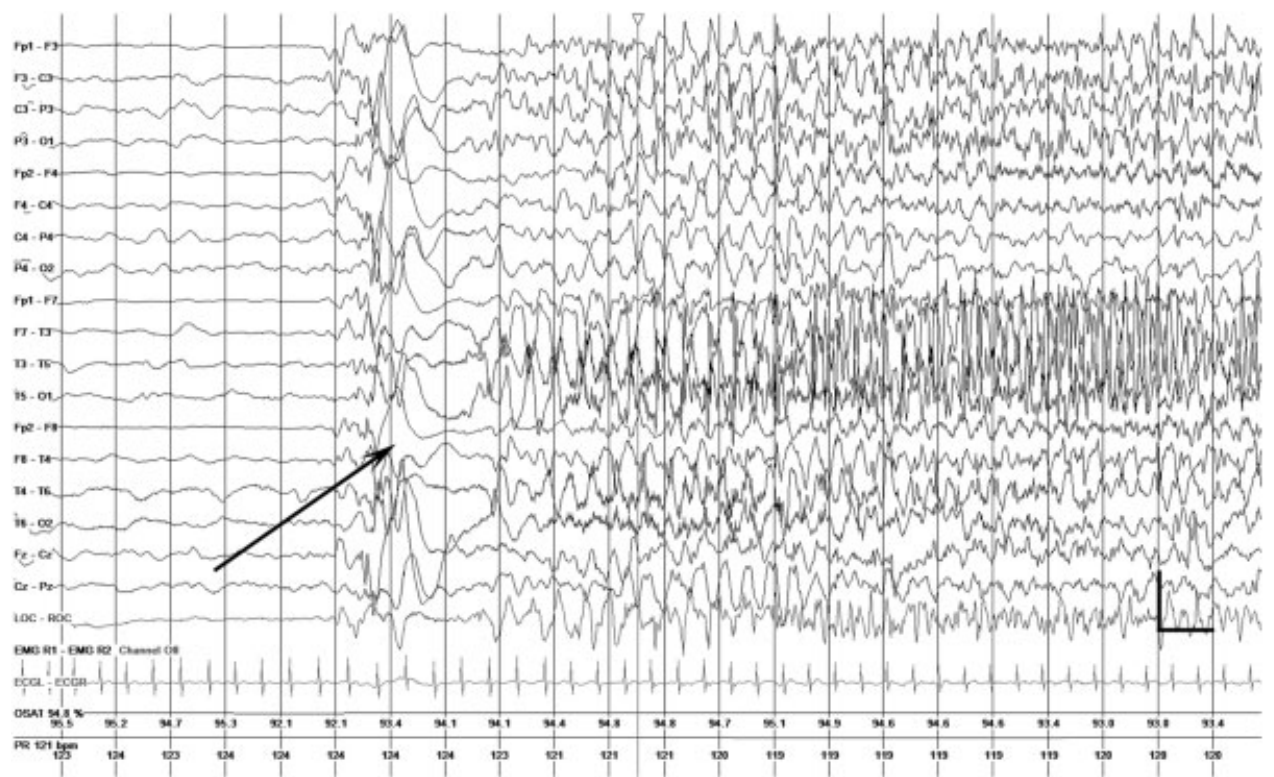

A

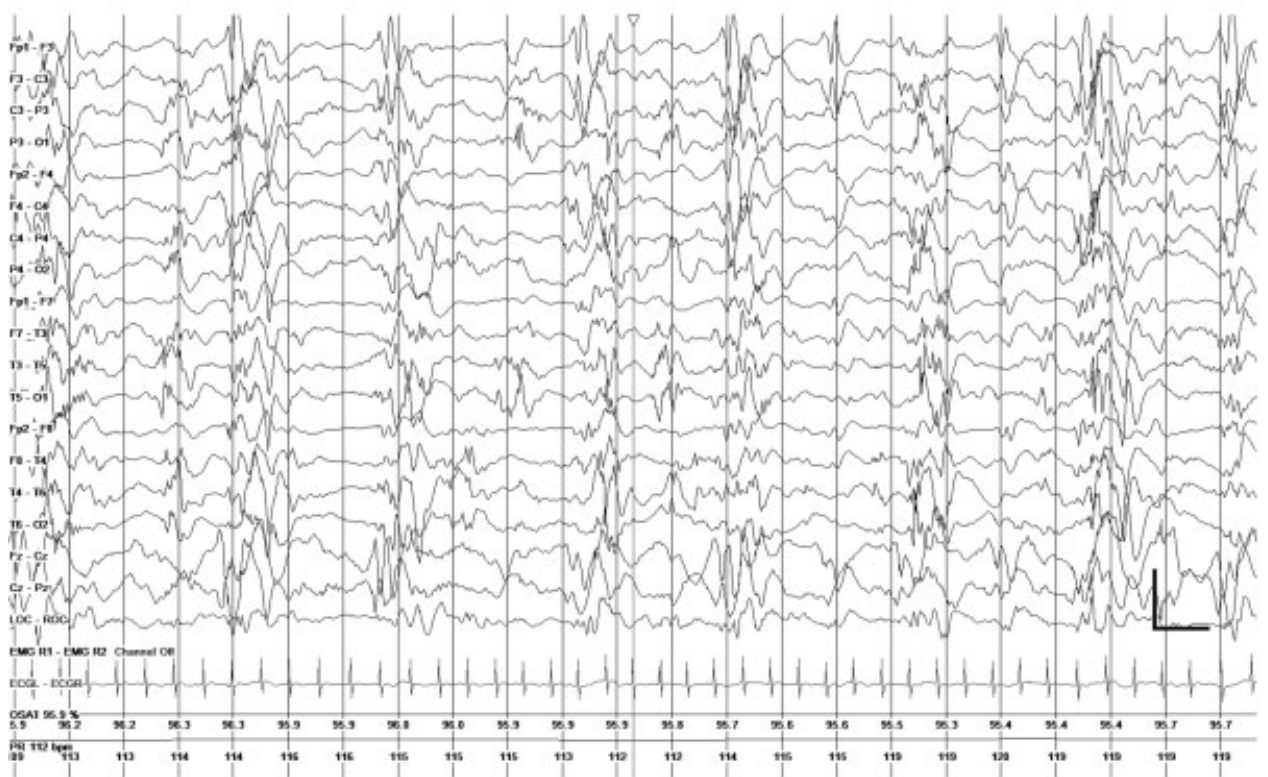

B

Fig. 2 (A) EEG shows ictal electrodecremental response (arrow) associated with an epileptic spasm. This was followed by repetitive spike discharges recoded maximal in the left temporal region at onset. The ictal semiology associated with the focal onset discharges was head and eye deviation to the right along with stiffening and flexion of the right arm. Vertical bar $300 \mu \mathrm{V}$. Horizontal bar $=2$ seconds. (B) A burst suppression variant of hypsarrhythmia recorded interictally. EEG, electroencephalography; EMG, electromyogram; PR. pulse rate.

panel of 66 genes, associated with early infantile epileptic encephalopathy and well-characterized syndromes where seizures are a predominant symptom, revealed a de novo, heterozygous, pathogenic variant in the MECP2 gene designated c.1079C > A. This variant, which was also confirmed with Sanger's sequencing and is predicted to result in a premature protein termination p.Ser $360^{*}$ (-Table 1). This specific mutation has previously been described as causative for Rett's syndrome ${ }^{5}$ but so far has not been associated with IS. The patient was also found to be heterozygous for a variant of uncertain significance in the SCN9A gene designated c.1895 G > A, which was predicted to result in an amino acid substitution p.Ser620Asn. This variant has been regis- tered in the EXAC database with an allele frequency of $1 / 120,700$. The amino acid residue at p.Ser620 of the SCN9A protein is highly conserved during evolution and the amino acid substitution programs PolyPhen-2, SIFT, and MutationTaster predict the p.Ser620Asn to be probably damaging, deleterious, and disease causing, respectively. Interestingly enough, the unaffected father was homozygous for this same variant in the SCN9A gene. No variants in CDKL5, FOXG1 genes were found.

The epileptic-spasms were initially refractory to prednisolone but eventually responded to a combination of vigabatrin and topiramate. However, the hypsarrhythmia recorded on multiple EEGs remained refractory to all treatments including 

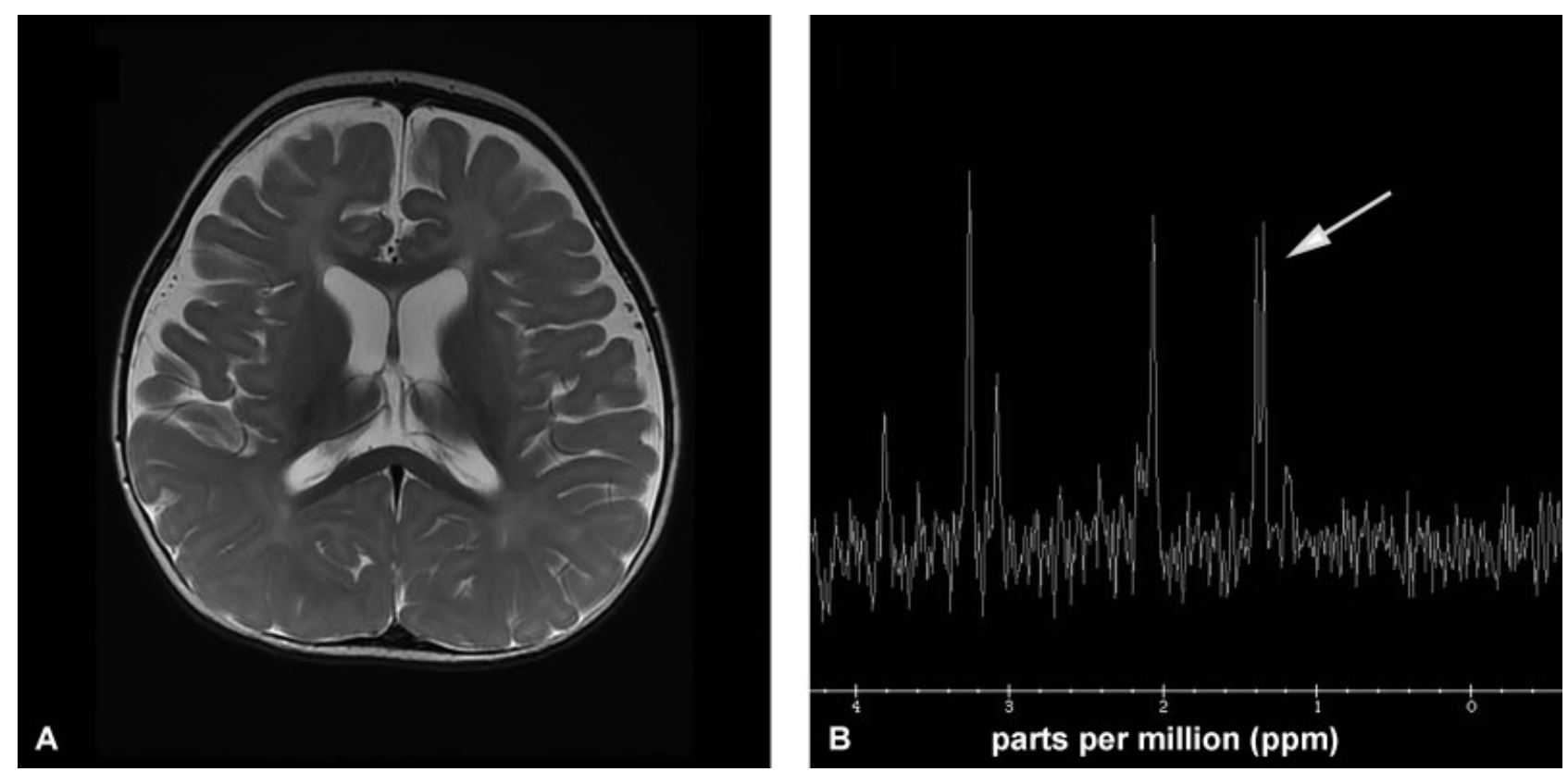

Fig. 3 (A) MRI brain; representative axial T-2 weighted image obtained from the patient at 9 months of age shows mild prominence of the ventricles and extra-axial CSF spaces. (B) Single voxel MR spectroscopy from the lateral ventricles shows a doublet peak at approximately 1.3 ppm suggestive of a lactate peak. However, MRS from the left basal ganglia showed no lactate peak (not shown). MRI, magnetic resonance imaging.

Table 1 Summary of the known genetic abnormalities identified in the patient with intractable infantile spasms

\begin{tabular}{|l|l|l|l|l|l|}
\hline Gene & DNA & Genotype & Predicted effect & Classification & Inheritance \\
\hline MECP2 & $1079 \mathrm{C}>\mathrm{A}$ & HET & pSer360* & Pathogenic & de novo \\
\hline SCN9A & $1859 \mathrm{G}>\mathrm{A}$ & HET & pSer620Asn & VUS & Father \\
\hline SCN5A & $1369 \mathrm{~T}>\mathrm{G}$ & HET & pSer457Ala & VUS & Mother \\
\hline
\end{tabular}

Abbreviations: HET, heterozygote; VUS, variant of undetermined significance.

the ketogenic diet. Initial evidence of deceleration in head growth was seen at 11 months ( $\mathbf{- F i g . ~ 1 ) . ~ T h e ~ p a t i e n t ~ m a d e ~ n o ~}$ further developmental gains and then at 15 months of age the spasms were re-emerged. No further interventions were attempted in accordance with the parents' wishes. At 19 months the patient developed a cardiomyopathy and was admitted to hospital. A transthoracic ECHO (echocardiography) showed a dilated globular left ventricle with a severely reduced systolic function. The left ventricle ejection fraction was 17 to $18 \%$. A selenium level of $0.88 \mu \mathrm{mol} / \mathrm{L}$ was obtained which was considered normal for age. ${ }^{6}$ EKG showed sinus tachycardia with a normal QTc of 438 milliseconds.

Given the finding of cardiomyopathy in this patient, a 134 next generation sequencing gene panel customized for arrhythmogenic right ventricular cardiomyopathy, dilated cardiomyopathy, hypertrophic cardiomyopathy, left ventricular non-compaction cardiomyopathy, Noonan syndrome and restrictive cardiomyopathy was pursued. A variant of uncertain significance in the SCN5A gene was identified, c.1369T > G, p.Ser457Ala. This variant has not been described in literature or mutation database (ClinVar, HGMD, Paralogue annotation database or inherited arrhythmia database). Additionally, there are no paralogue variants mapped to SCN5A residue 457. There was one reported variant in the neighboring codon, p.Arg458Cys, detected in patients with Sudden Infant Death Syndrome (SIDS). However, it is reported that the variant is not a well conserved residue and there are 35 heterozygous carriers among 125,000 individuals in GnomAD reference population and one homozygote, indicating that this variant is unlikely to be disease-causing. The unaffected mother underwent familial variant testing and the same variant in the SCN5A gene was identified in her. The mother's electrocardiogram (EKG) and cardiac ECHO were normal. EKG performed on the father was normal. A cardiac ECHO was not performed on the father. The patient subsequently died due to complications related to the dilated cardiomyopathy.

\section{Discussion}

To our knowledge, this is one of two cases of IS that is confirmed due to MECP2 gene mutation. Rett's syndrome is an X-linked neuro-developmental disorder diagnosed typically in girls. ${ }^{7}$ Patients with Rett's syndrome usually present with language regression, gait abnormalities, loss purposeful hand movements, and the emergence of hand stereotypies. ${ }^{8}$ Necessary to the diagnosis of Rett's syndrome is a period of 
regression that our patient did not have but her emerging deceleration in head growth is typical for this disorder. Other distinct variant forms of Rett's syndrome have been well described which includes congenital (Rolando), early seizure (Hanefeld), and the preserved language (Zappella) variants. ${ }^{8}$ Our patient most closely fits the Hanefeld's variant in which IS are a prominent feature.

Epilepsy has been reported to occur in 50 to $90 \%$ of patients with Rett's syndrome. ${ }^{9}$ Multiple types of seizures have been described which include both focal impaired aware and generalized bilateral convulsive, myoclonic, and tonic seizures. Seizures are usually most prominent between 2 to 10 years of age during the plateau phase of this disease. ${ }^{9}$ Although it is suspected in the literature that some of these patients with IS may have a mutation in MECP2, it commonly turns out not to be the case, and instead mutations in CDKL5 are typically described. ${ }^{10,11}$ For instance, in one study describing a cohort of 183 patients with early onset epilepsy of which 30 had unexplained IS, five patients had mutations in CDKL5 and the remainder remained genetically undiagnosed. ${ }^{10}$ In that study, all of the patients were ruled out for mutations in MECP2. In another study, which described a cohort of 31 patients with Rett's syndrome of which $77.4 \%$ had a mutation in the MECP2 gene, none of the patients had IS. ${ }^{12}$ However, one patient with nucleotide changes (1364-1365ins C) in MECP2 presented with psychomotor regression and severe early onset epilepsy at 10 months and another patient with a single nucleotide polymorphism $(582 \mathrm{C}>\mathrm{T})$ was described as having deceleration of the head growth and developed the Rett's phenotype following acute epileptic encephalopathy. ${ }^{12}$ Although both these patients had early onset epilepsy it was not reported as to whether these patients had IS. Our patient had a mutation in the MECP2 gene for a variant designated c.1079C > A which so far has not been reported to be associated with epilepsy or with IS in the literature. ${ }^{5}$ Additionally, results derived from 1,248 patients with MECP2-related Rett's syndrome obtained from the Rett's syndrome network database showed that $61 \%$ had epilepsy and none were reported to have IS. ${ }^{13}$ In that study, patients with genes, such as CDKL5 and FOXG1, were known to be associated with variant Rett's syndrome, as well as patients with MECP2 duplication were excluded which may explain why IS was not reported. ${ }^{13}$ Interestingly, late onset epileptic spasms in contrast to our case have been reported in boys with MECP2 duplication related Rett's syndrome, with a mean age of seizure onset of 6 years. ${ }^{4}$ The seizures in patients with late onset epileptic spams are usually intractable to treatment. Note that IS were described in the original publication of Hanefield's variant Rett's but a mutation in the MECP2 gene was not confirmed as the genetic cause of Rett's syndrome was unknown at that time and genetic testing was generally unavailable. ${ }^{14}$ To our knowledge, just one case of possible of IS in a girl harboring a missense mutation in MECP2 (S388P) was published by Conforti et al. ${ }^{15}$ However, the only details given were that the patient had severe developmental delay in the first month of life, IS at 5 months and renal failure at 5 years.

Our patient also had variants in the SCN9A and SCN5A genes. In the literature, mutations in the $S C N 9 A$ gene are known to cause primary erythromelalgia and febrile seizures, although people having a mutation in the SCN9A gene are usually asymptomatic. ${ }^{16}$ Mutations in the SCN9A gene have also been associated with a worse prognosis in patients with Dravet's syndrome harboring the SCN1A mutation. ${ }^{16}$ In our case, the SCN9A mutation is likely not the primary disease-causing mutation, as it was present in the unaffected father. There is always the possibility, that this SCN9A variant may have altered the phenotype of our patient but this remains unconfirmed. The SCN5A gene encodes a cardiac sodium channel and it is abundant in the myocardium but not in the cerebral cortex. ${ }^{17}$ Since it is not expressed in the brain, it is unlikely to contribute to the epileptic phenotype. Mutations in SCN5A have been associated with Brugada's syndrome, prolonged QT syndrome, dilated cardiomyopathy, familial atrial fibrillation, nonprogressive and progressive heart block, sick sinus syndrome, and familial ventricular fibrillation. ${ }^{16,17}$ Given the association with dilated cardiomyopathy, the mutation in the SCN5A gene, which was found in this patient, may have accounted for the cardiomyopathy but this remains speculative, especially as the variant was inherited from the unaffected mother. As whole exome or genome wide sequencing was not done in our patient it is also unknown whether other unidentified genetic variations have contributed to the severe phenotype in our patient.

\section{Conclusion}

In summary, mutations in the MECP2 gene can cause IS, but very rarely and may require the presence of other mutations in other unidentified genes. We can conclude that broad genetic panels, whole exome or genome wide sequencing may be needed in diseases such as MECP2-reated disorders with atypical presentations to better understand the disease etiology. More work is required to determine the harmful or potential beneficial effect of variant of uncertain significance in the presence of known pathogenic mutations.

Funding

None.

Conflict of Interest

None declared.

\section{References}

1 Karvelas G, Lortie A, Scantlebury MH, Duy PT, Cossette P, Carmant L. A retrospective study on aetiology based outcome of infantile spasms. Seizure 2009;18(03):197-201

2 Zhao Y, Zhang X, Bao X, et al. Clinical features and gene mutational spectrum of CDKL5-related diseases in a cohort of Chinese patients. BMC Med Genet 2014;15:24

3 Paciorkowski AR, Thio LL, Dobyns WB. Genetic and biologic classification of infantile spasms. Pediatr Neurol 2011;45(06): 355-367

4 Caumes R, Boespflug-Tanguy O, Villeneuve N, et al. Late onset epileptic spasms is frequent in MECP2 gene duplication: electroclinical features and long-term follow-up of 8 epilepsy patients. Eur J Paediatr Neurol 2014;18(04):475-481 
5 Buyse IM, Fang P, Hoon KT, Amir RE, Zoghbi HY, Roa BB. Diagnostic testing for Rett syndrome by DHPLC and direct sequencing analysis of the MECP2 gene: identification of several novel mutations and polymorphisms. Am J Hum Genet 2000;67(06): 1428-1436

6 Muntau AC, Streiter M, Kappler M, et al. Age-related reference values for serum selenium concentrations in infants and children. Clin Chem 2002;48(03):555-560

7 Rett A. On a unusual brain atrophy syndrome in hyperammonemia in childhood [article in German]. Wien Med Wochenschr 1966;116(37):723-726

8 Neul JL, Kaufmann WE, Glaze DG, et al; RettSearch Consortium. Rett syndrome: revised diagnostic criteria and nomenclature. Ann Neurol 2010;68(06):944-950

9 Dolce A, Ben-Zeev B, Naidu S, Kossoff EH. Rett syndrome and epilepsy: an update for child neurologists. Pediatr Neurol 2013;48 (05):337-345

10 Bahi-Buisson N, Nectoux J, Rosas-Vargas H, et al. Key clinical features to identify girls with CDKL5 mutations. Brain 2008;131 (Pt. 10):2647-2661
11 Nissenkorn A, Gak E, Vecsler M, Reznik H, Menascu S, Ben Zeev B. Epilepsy in Rett syndrome-the experience of a National Rett Center. Epilepsia 2010;51(07):1252-1258

12 Huppke P, Laccone F, Krämer N, Engel W, Hanefeld F. Rett syndrome: analysis of MECP2 and clinical characterization of 31 patients. Hum Mol Genet 2000;9(09):1369-1375

13 Nissenkorn A, Levy-Drummer RS, Bondi O, et al. Epilepsy in Rett syndrome-lessons from the Rett networked database. Epilepsia 2015;56(04):569-576

14 Hanefeld F. The clinical pattern of the Rett syndrome. Brain Dev 1985;7(03):320-325

15 Conforti FL, Mazzei R, Magariello A, et al. Mutation analysis of the MECP2 gene in patients with Rett syndrome. Am J Med Genet A 2003;117A(02):184-187

16 Singh NA, Pappas C, Dahle EJ, et al. A role of SCN9A in human epilepsies, as a cause of febrile seizures and as a potential modifier of Dravet syndrome. PLoS Genet 2009;5(09):e1000649

17 Goldman AM, Behr ER, Semsarian C, Bagnall RD, Sisodiya S, Cooper PN. Sudden unexpected death in epilepsy genetics: molecular diagnostics and prevention. Epilepsia 2016;57(Suppl 1):17-25 